could be used only by women, and the services list was extended with special services for the women that were selected according to the results of a focus group. The additional services were informational classes with a psychologist, lawyer, social worker, individual consultations with a therapist, infectious disease specialist, gynaecologist, etc., an opportunity to participate in the Creative studio, manicure and haircut, a self-aid group, food and hygienic kits for women and children. The services were provided by female specialists only, including the use of the "Equal to Equal" method. At the meetings, information regarding to the women's health and rights was brought to the clients so that they were able to feel more confident and based their choices on better informed decisions.

From May to October of 2012, more than 300 female drug consumers were involved in the project's services. Among them, a volunteer group ( 9 women) was prepared for the future prophylactic work with women. The project's activity demonstrated its actuality and the need for it to be continued. During the project implementation the participation of female drug consumers reached $32 \%$.

\section{P3.396 ARE THE WORLD AIDS CONFERENCES GUILTY OF GENDER BIAS? EVIDENCE FROM TRENDS IN THE MONITORING OF WAC SCIENTIFIC DISCOURSE FROM 1989 TO 2012}

doi:10.1136/sextrans-2013-051184.0849

'B E Spencer, ${ }^{2} \mathrm{~A}$ Jeannin, ${ }^{2} \mathrm{~F}$ Dubois-Arber, ${ }^{3 \mathrm{P}}$ Iriarte. ${ }^{1}$ Institute of Social and Preventive Medicine (IUMSP), Lausanne University Hospital, Lausanne, Switzerland; 'Institute of Social and Preventive Medicine (IUMSP), Lausanne University Hospital, Lausanne, Switzerland; 'Public Health Documentation Centre (CDSP), Lausanne University Hospital, Lausanne, Switzerland

Background The issue of gender is acknowledged as a key issue for the AIDS epidemic. World AIDS Conferences (WAC) have constituted a major discursive space for the epidemic. We sought to establish the balance regarding gender in the AIDS scientific discourse by following its development in the published proceedings of WAC. Fifteen successive WAC 1989-2012 served to establish a "barometer" of scientific interest in heterosexual and homo/bisexual men and women throughout the epidemic. It was hypothesised that, as in other domains of Sexual and Reproductive Health, heterosexual men would be "forgotten" partners.

Method Abstracts from each conference were entered in electronic form into an Access database. Queries were created to generate five categories of interest and to monitor their annual frequency. All abstract titles including the term "men" or "women" were identified. Collections of synonyms were systematically and iteratively developed in order to classify further abstracts according to whether they included terms referring to "homo/bisexual" or "heterosexual". Reference to "Mother to Child Transmission" (MTCT) was also flagged. Results The category including "men", but without additional reference to "homo-bisexuel" (i.e. referring to men in general and/or to heterosexual men) consistently appears four times less often than the equivalent category for women. Excluding abstracts on women and MTCT has little impact on this difference. Abstracts including reference to both "men" and "homo-bisexual" emerge as the secondmost frequent category; presence of the equivalent category for women is minimal.

Conclusion The hypothesised absence of heterosexual men in the AIDS discourse was confirmed. Although the relative presence of homo-bisexual men and women as a focal subject may be explained by epidemiological data, this is not so in the case of heterosexual men and women. This imbalance has consequences for HIV prevention.

\section{P3.397 PREVENTION SERVICES, HEALTH-SEEKING BEHAVIOURS, AND STI PREVALENCE AMONG FEMALE ENTERTAINMENT WORKERS IN CAMBODIA}

doi:10.1136/sextrans-2013-051184.0850
'W P Killam, ${ }^{2} \mathrm{P}$ Mun, ${ }^{3} \mathbf{J}$ D Mutuc, ${ }^{2} \mathrm{~N}$ Chann, ${ }^{4} \mathrm{~S}$ Chan, ${ }^{1} \mathrm{R}$ W Shiraishi, ${ }^{1} \mathrm{D}$ Warren, ${ }^{2} \mathrm{M}$ C Vun. 'Centers for Disease Control and Prevention, Center for Global Health, Atlanta, GA, United States, ${ }^{2}$ National Center for HIVIAIDS, Dermatology and STDs, Ministry of Health, Phnom Penh, Cambodia, ${ }^{3}$ Association of Schools of Public Health/Centers for Disease Control and Prevention Allan Rosenfield Global Health Fellow, Phnom Penh, Cambodia, ${ }^{4}$ US Centers for Disease Control and Prevention, Cambodia Country Office, Phnom Penh, Cambodia

Background Cambodia's brothel-based prevention programmes successfully promoted condom use among female sex workers since 1994. HIV and sexually transmitted infection (STI) prevalence decreased precipitously from 1998 to 2006. In 2008 brothels became illegal, and entertainment venues (karaoke bars, beer gardens and massage parlours) increased rapidly. Many female entertainment workers (FEWs) at these venues provide transactional sex. We explored receipt of prevention services and associated knowledge, health-seeking behaviour, and prevalence of STIs and HIV among Cambodian FEWs.

Methods In 2011, a stratified multi-stage cluster survey was conducted among 2,564 FEWs in selected provinces of Cambodia. Voluntary, anonymous interviews collected information on receipt of prevention services and sexual behaviours. Biological testing was conducted for HIV, syphilis, gonorrhoea, and chlamydia. Analyses were weighted and controlled for the design of the survey. Logistic regression models were used to examine associations between interventions, health-seeking behaviours, and STIs.

Results Prevalence of any STI and HIV was $20.8 \%$ and $2.6 \%$, respectively. Among FEWs, 86.9\% reported receiving HIV/STI education, most commonly from an outreach worker $(47.7 \%)$ or television (46.1\%). Receipt of a prevention message was associated with getting an HIV test (odds ratio [OR] $=2.6,95 \%$ confidence interval [CI]: 1.8-3.6) and sexual health check-up (OR $=2.6,95 \%$ CI: $1.7-$ 3.9 ) in the last 12 months, and higher HIV knowledge ( $O R=1.7$, 95\% CI: 1.1-2.8). Condom provision was also associated with health-seeking behaviours. Receipt of prevention interventions was not associated with reported recent condom usage or STIs, although further analyses are ongoing in order to adjust for potential confounders.

Conclusion The rapid expansion of non-brothel-based entertainment work challenges prevention efforts among Cambodian FEW, who have high STI prevalence and risk behaviours. Prevention education and condom provision are associated with increased knowledge and health seeking behaviours, but not self-reported condom use or reduced STI prevalence in this cross-sectional snapshot.

\section{P3.398 SENTINEL SURVEILLANCE AND PREVENTION OF SEXUALLY TRANSMITTED INFECTIONS AMONG FEMALE SEX WORKERS IN GUATEMALA: FIRST FINDINGS FROM VICITS}

doi:10.1136/sextrans-2013-051184.0851

C A Galindo Arandi, I Loya, J Jacobson, F Arana, S Morales Miranda. Universidad de/ Valle de Guatemala, Guatemala, Guatemala

Background In Guatemala, female sex workers (FSWs) have historically demonstrated a high HIV prevalence and are considered a key group in heterosexual HIV transmission. A system for sentinel surveillance of HIV-STI infection, risk behaviours and STI control targeting FSW, incorporating prevention components (VICITS) was rolled at public health centres in four Guatemalan cities, beginning in 2007, 2008, 2010 and 2011, respectively. We present the first assessment of scale-up, retention and HIV-STI infection trends among VICITS users.

Methods The number of FSW accessing VICITS each year in 2007-2011 was estimated by health centre based on a unique, person-level identifier. Retention was estimated as the percent of new users accessing VICITS in a given year who returned for at least one control visit. Percent of users infected was estimated from regular, 